\title{
Estratégia para o tratamento conservador da camptodactilia de tipo III em criança com síndrome de Beals-Hecht*
}

\section{Strategy for the Conservative Treatment of Type-III Camptodactyly in Children with Beals-Hecht Syndrome}

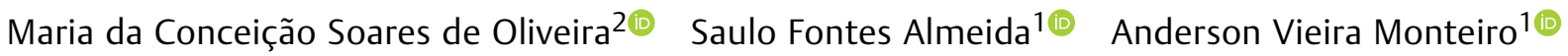 \\ Maria Caroliny Soares de Oliveira ${ }^{3(0)}$ Felipe Soares Figueiredo ${ }^{3}$ () Diego Pinheiro Aguiar $^{3,4}$
}

\footnotetext{
${ }^{1}$ Centro de Cirurgia da Mão, Instituto Nacional de Traumatologia e Ortopedia, Rio de Janeiro, RJ, Brasil

2 Área de Reabilitação, Instituto Nacional de Traumatologia e Ortopedia, Rio de Janeiro, RJ, Brasil

${ }^{3}$ Divisão de Pesquisa, Instituto Nacional de Traumatologia e Ortopedia, Rio de Janeiro, RJ, Brasil

${ }^{4}$ Laboratório de Biomodelos e Prototipagem, Centro Universitário Estadual da Zona Oeste, Rio de Janeiro, RJ, Brasil
}

\author{
Endereço para correspondência Maria da Conceição Soares de \\ Oliveira, Avenida Brasil, 500, Caju, Rio de Janeiro, RJ, 20940-070, Brasil \\ (e-mail: mcsoaresto@gmail.com).
}

Rev Bras Ortop

\section{Resumo \\ Palavras-chave \\ - articulações dos dedos \\ - deformidades congênitas da mão \\ - órteses \\ - reabilitação}

Os autores apresentam um caso bem-sucedido no tratamento conservador da camptodactilia de tipo III em paciente com síndrome de Beals-Hecht. A camptodactilia é uma deformidade em flexão da articulação interfalangeana proximal (IFP), no sentido anteroposterior, indolor, e bilateral em 2/3 dos casos. A de tipo III é a forma mais grave e incapacitante, pois geralmente acomete vários dedos e está associada a síndromes e outras malformações. O caso apresentado teve a correção alcançada com o uso sistemático de órteses estáticas iniciado aos 7 meses de idade e concluído após 23 meses e meio de intervenção.

The authors present a successful case in the conservative treatment of type-III camptodactyly in a patient with Beals-Hecht syndrome. Camptodactyly is a flexion deformity of the proximal interphalangeal (PIP) joint, in the anteroposterior direction, painless and bilateral in 2/3 of the cases. Type-III is the most severe and disabling form, as it usually affects several fingers and is associated with syndromes and other malformations. The case herein reported had the correction achieved with the systematic use of static orthoses started at 7 months of age and completed after 23 and a half months of the intervention.

Trabalho desenvolvido na Divisão de Pesquisa, Instituto Nacional de

Traumatologia e Ortopedia Jamil Haddad, Rio de Janeiro, RJ, Brasil.

recebido

09 de Novembro de 2020

aceito

08 de Março de 2021
DOI https://doi.org/

$10.1055 / \mathrm{s}-0041-1739401$. ISSN 0102-3616. (c) 2021. Sociedade Brasileira de Ortopedia e Traumatologia. All rights reserved.

This is an open access article published by Thieme under the terms of the Creative Commons Attribution-NonDerivative-NonCommercial-License, permitting copying and reproduction so long as the original work is given appropriate credit. Contents may not be used for commercial purposes, or adapted, remixed, transformed or built upon. (https://creativecommons.org/ licenses/by-nc-nd/4.0/)

Thieme Revinter Publicações Ltda., Rua do Matoso 170, Rio de Janeiro, RJ, CEP 20270-135, Brazil 


\section{Introdução}

A camptodactilia é uma deformidade em flexão da articulação interfalangeana proximal (IFP), de origem congênita e não traumática. É uma condição rara, com prevalência em torno de $1 \%$, sendo o quinto dedo o mais afetado. ${ }^{1}$ É classificada em tipos I, II e III. Tipo I: camptodactilia infantil, que acomete geralmente o dedo mínimo de forma isolada. Tipo II: camptodactilia da adolescência, de rápida evolução com o estirão do crescimento. Tipo III: presente ao nascimento, acomete vários dedos, e está associada a outras síndromes. A camptodactilia acomete estruturas que cruzam a articulação, e elas estão implicadas como uma das possíveis causas. Podem ser encontradas alterações na pele, aponeurose, tendões, músculo lumbrical, músculo flexor superficial dos dedos, e ligamentos. ${ }^{1}$ O tratamento inicial é o conservador, com o uso de órteses e alongamentos passivos, ou o cirúrgico. ${ }^{2}$ Em casos em que a flexão da articulação IFP é $\geq a$ a $60^{\circ}$, a indicação é cirúrgica. ${ }^{3,4}$

A síndrome de Beals-Hecht é uma síndrome rara, diagnosticada em menos de 1 a cada 10 mil pacientes no mundo. ${ }^{5,6} \mathrm{O}$ quadro clínico consiste em contraturas congênitas de múltiplas articulações, ${ }^{7}$ membros longos e delgados, aracnodactilia contratural congênita, cifoescoliose, e anomalias dos pavilhões auriculares. ${ }^{3}$ As contraturas podem reduzir de gravidade, mas a camptodactilia presente nos dedos persiste. $^{7}$

\section{Relato de Caso}

Paciente masculino, de 6 meses de idade, branco, atendido no Ambulatório de Cirurgia da Mão de nossa instituição pela primeira vez em outubro de 2013. Observou-se a presença de alguns sinais clínicos, a saber: flexão das articulações IFPs dos dedos médio, anelar e mínimo, flexão de punhos, e hiperextensão das articulações metacarpofalangeanas (MCFs) bilateralmente, alterações em pavilhão auricular, e pectus excavatum. Todas as contraturas presentes eram rígidas. 0 paciente foi diagnosticado com camptodactilia de tipo III, e optou-se pelo tratamento conservador com ortetização em novembro de 2013. Não foi feita a goniometria inicial do paciente devido à dificuldade de execução, mas fez-se o registro fotográfico (-Figura 1A-E). As contraturas em flexão das articulações IFPs apresentavam mais de $90^{\circ}$. 0 uso de órteses estáticas inicialmente foi feito de forma ininterrupta, ${ }^{8}$ e os retornos se deram a cada três meses, para acompanhamento e ajustes ou trocas de órteses.

O processo de ortetização teve seu início aos sete meses de idade. $O$ objetivo inicial foi chegar à posição em neutro da flexão da articulação IFP, da flexão do punho, e da
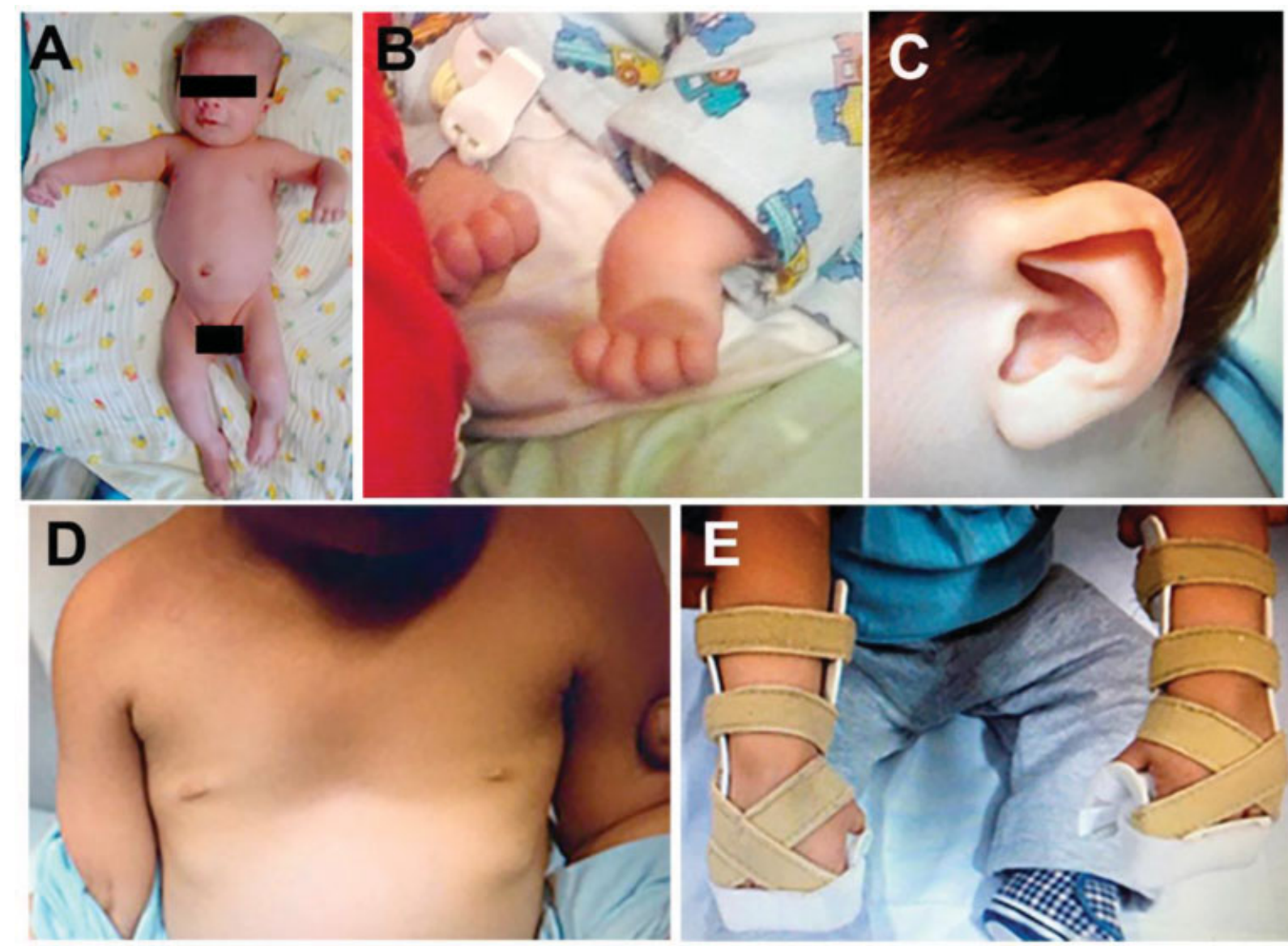

Fig. 1 Características fenotípicas do paciente com Síndrome de Beals Heacht. Paciente com 2 meses (A), deformidades no punho, nas articulações metacarpofalangeanas e inferfalangeanas (B), anomalias no pavilhão auricular (C), pectus excavatum (D), e órtese de posicionamento com apoio dorsal (E). 

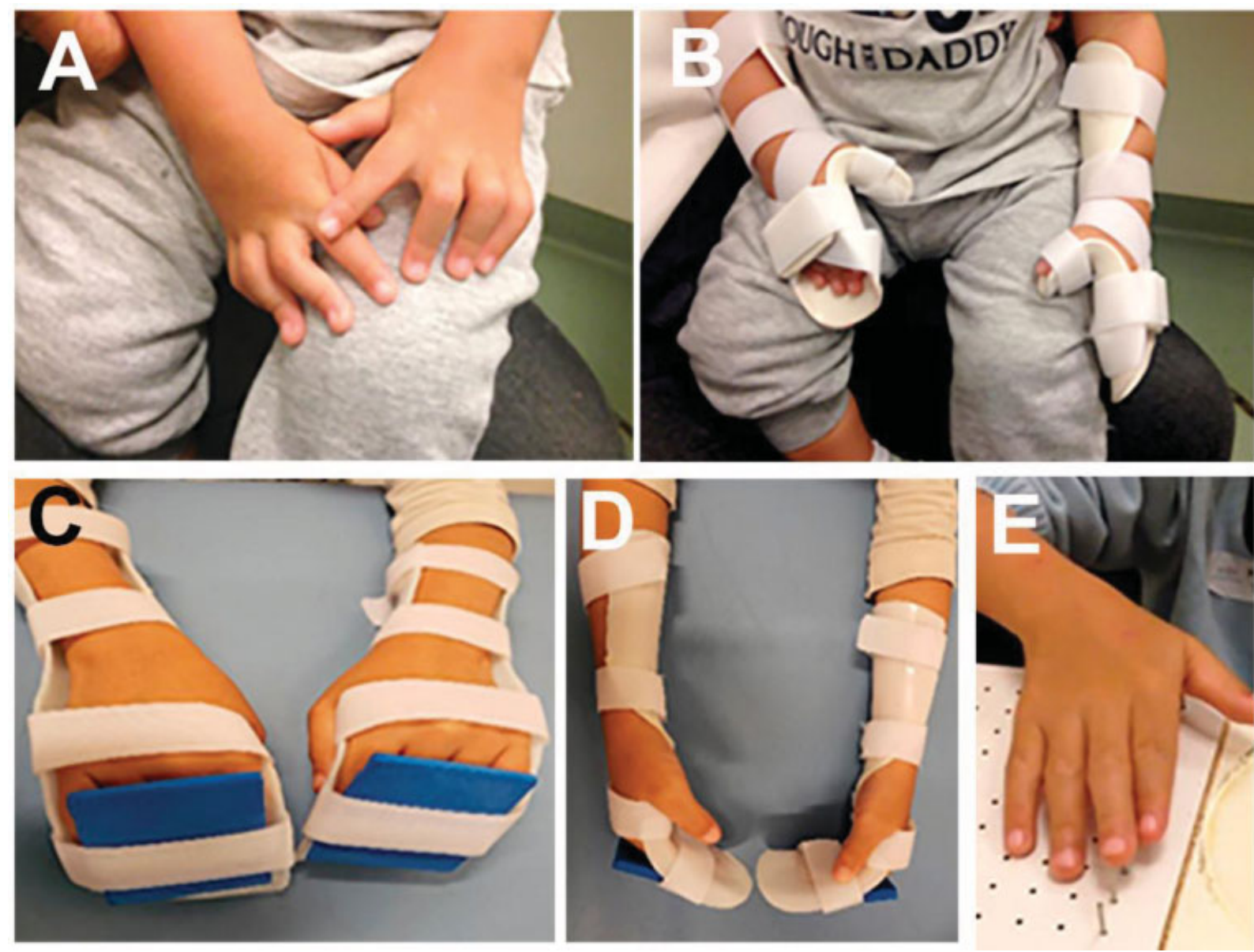

Fig. 2 Demonstração da evolução do tratamento conservador do paciente com camptodactilia. Início da correção (A), órteses de posicionamento com manutenção do apoio dorsal (B), órteses de uso noturno com polegares livres e placa de acetato-vinilo de etileno (EVA) na região dorsal de dedos (C), órteses de uso noturno em vista lateral (D), e extensão completa de dedos (E).

hiperextensão da articulação MCF, alcançada em fevereiro de 2015 (-Figura 2 A-E).

Após alcançar a posição em neutro, a órtese foi modificada, iniciando com uma leve flexão da articulação MCF e dispositivo de apoio dorsal seguindo a angulação da órtese, para exercer pressão nos dedos contra a órtese, mantendo assim a posição, além de auxiliar a flexão da articulação MCF. A partir de julho de 2015, já com 26 meses, as órteses passaram a ser confeccionadas com os polegares livres, as articulações MCF fletidas, e o punho em extensão (-Figura 2, A-D).
O uso das órteses passou a ser feito apenas no período noturno, em torno de 8 horas, a partir junho de 2016. Foi alcançada a correção da camptodactilia (- Figura 2, E e - Figura 3 A-C).

\section{Discussão}

Neste caso de camptodactilia de tipo III rígida em criança com síndrome de Beals Heacht, a intervenção precoce ${ }^{9}$ com órteses estáticas proporcionou desfecho satisfatório, com a
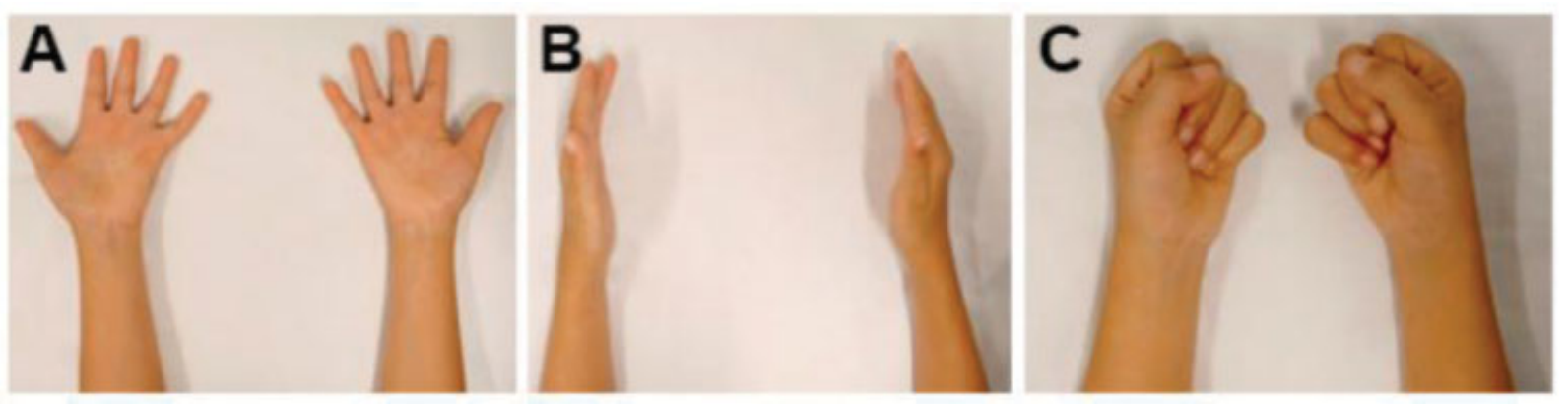

Fig. 3 Morfologia do membro superior após o tratamento conservador por 23 meses e meio. Posição supina com dedos em extensão (A), vista lateral (B), e dedos fletidos (C). 
regressão da deformidade e a obtenção do uso funcional das mãos. O uso de órteses deve ser a primeira escolha para o início do tratamento, inclusive nos casos sindrômicos. ${ }^{9}$

A multiplicidade de estruturas envolvidas na camptodactilia que são responsáveis pelo desequilíbrio articular podem chegar a um equilíbrio com a remodelagem tecidual por meio de órteses. ${ }^{9}$

A utilização de órteses para a remodelação do tecido musculoesquelético é uma opção de baixa complexidade; porém, é necessário um período de uso relativamente longo para que a remodelação ocorra. ${ }^{10}$

O uso do dispositivo de apoio dorsal otimizou o alongamento das estruturas responsáveis pela contratura em flexão na articulação IFP.

O acompanhamento clínico do paciente em questão será mantido até o final do crescimento esquelético, para evitar possível recidiva de contratura de partes moles. ${ }^{4,8}$

O envolvimento da família e sua colaboração foi um diferencial neste tratamento conservador. A intervenção é relativamente longa e exaustiva, mas é necessária. ${ }^{10}$

\section{Suporte Financeiro}

Não houve suporte financeiro de fontes públicas, comerciais, ou sem fins lucrativos.

Conflito de interesses

Os autores declaram não haver conflito de interesses.

\section{Referências}

1 Salazard B, Quilici V, Samson P. [Camptodactyly]. Chir Main 2008; 27(Suppl 1):S157-S164

2 Wang AMQ, Kim M, Ho ES, Davidge KM. Surgery and Conservative Management of Camptodactyly in Pediatric Patients: A Systematic Review. Hand (N Y) 2019;15(06): 761-770

3 Evans BT, Waters PM, Bae DS. Early Results of Surgical Management of Camptodactyly. J Pediatr Orthop 2017;37(05): e317-e320

4 Almeida SF, Monteiro AV, Lanes RCS. Evaluation of treatment for camptodactyly: retrospective analysis on 40 fingers. Rev Bras Ortop 2014;49(02):134-139

5 Jurko A Jr, Krsiakova J, Minarik M, Tonhajzerova I. Congenital contractural arachnodactyly (Beals-Hecht syndrome): a rare connective tissue disorder. Wien Klin Wochenschr 2013;125(910):288-290

6 Tunçbilek E, Alanay Y. Congenital contractural arachnodactyly (Beals syndrome). Orphanet J Rare Dis 2006;1:20

7 Meena JP, Gupta A, Mishra D, Juneja M. Beals-Hecht syndrome (congenital contractural arachnodactyly) with additional craniospinal abnormality: a case report. J Pediatr Orthop B 2015; 24(03):226-229

8 Hori M, Nakamura R, Inoue G, et al. Nonoperative treatment of camptodactyly. J Hand Surg Am 1987;12(06):1061-1065

9 Benson LS, Waters PM, Kamil NI, Simmons BP, Upton J 3rd. Camptodactyly: classification and results of nonoperative treatment. J Pediatr Orthop 1994;14(06):814-819

10 Lethbridge $\mathrm{K}$, Wollin L. A review of conservative management of camptodactyly in children and adolescent. Hand Ther 2014;19 (04):93-101 